\title{
Behavioural response to pain in healthy neonates
}

\author{
J Alison Rushforth, Malcolm I Levene
}

\begin{abstract}
A bedside technique for evaluating the behavioural response of healthy neonates to pain was assessed. Thirty six term infants (median gestational age 40 weeks; median postnatal age 4 days) and 31 preterm infants (median gestational age 34 weeks; median postnatal age 4 days) were assessed at the cotside for their response to heel preparation and heel lance for routine blood sampling. The facial actions of brow bulge, eye squeeze, nasolabial furrow, and open mouth were noted, and also the presence or absence of crying.
\end{abstract}

Thirty five (97\%) term and 26 (84\%) preterm infants showed an increase in the number of behaviours in response to heel lance. Brow bulge and nasolabial furrow were seen most often, and occurred more often than crying in the two groups. There was good interobserver agreement $(94 \%)$.

The consistency of response and the high degree of interobserver agreement makes this method of behavioural assessment of acute pain of use in healthy neonates.

(Arch Dis Child 1994; 70: F174-F176)

Pain is a subjective experience and to measure it in an objective way is difficult. Infants have been reported to be capable of experiencing pain irrespective of gestational age, ${ }^{1}$ but the response to pain may differ with gestational age. ${ }^{2} 3$ By using video recordings, Grunau and Craig showed that most $(96 \%)$ term neonates responded consistently to a painful stimulus with a cluster of facial actions, namely brow bulge, eye squeeze, nasolabial furrow, and open mouth. ${ }^{4}$ These facial actions are also seen more often in response to painful invasive procedures such as intramuscular injections than in response to non-invasive procedures such as alcohol swabbing of the thigh or application of triple dye to the umbilicus. ${ }^{5}$

It has been shown that there is a good correlation between observers scoring neonatal facial actions occurring in response to painful stimuli when analysed in playback from video recordings. ${ }^{6}$ This suggests that facial actions may be a useful tool in pain assessment in a clinical setting, and also as a measure of pain in interventional studies - for example, the use of analgesia or anaesthesia before painful procedures.

In adults the facial actions associated with pain have been shown to be remarkably consistent across different pain modalities - for example, pressure, ischaemia, cold, and electric shock. This has led researchers to suggest that observations in adults could be restricted to the more common facial actions seen in response to pain, namely brow lowering, lid tightening and cheek raising, nose wrinkling and lip raising, and eye closure. ${ }^{7}$ These facial actions are remarkably similar to the four most commonly seen in neonates in response to pain.

We were interested to see if simple bedside observation of infants during heel stab blood tests was as reliable as the use of video recordings in the detection of behavioural responses to painful stimuli.

\section{Subjects and methods}

Healthy newborn infants requiring routine heel stab blood tests for measurement of blood glucose or bilirubin, or for biochemical screening, were recruited to the study. All blood samples were taken as part of the infants' routine care. The infant's mother was always present during blood sampling and consent was obtained from her before the observations were made.

The infant's state of arousal was assessed before heelstab, as described by Prechtl and Beintema ${ }^{8}: 1=$ eyes closed, regular respiration, no movement; $2=$ eyes closed, irregular respiration, gross movements; 3 =eyes open, no gross movements; $4=$ eyes open, continual gross movements, no crying; $5=$ eyes open or closed, fussing, or crying.

Four items of facial action (brow bulge, eye squeeze, nasolabial furrow, and open mouth) and the presence or absence of crying were used as a measure of response to external stimulation. These responses were assessed once during heel preparation (non-painful stimulus) and again after heel lancing (painful stimulus). Each response was given a score of 1 if present and 0 if absent, so that a total score ranging from 0 to 5 was possible. This was termed the control score if it represented the response to heel preparation and the pain score if it represented the response to heel lance. The reaction to heel squeeze was not assessed as the length of this phase varied with the volume of blood required, and we expected the infant's behavioural response to reflect the duration of heel squeeze.

Facial actions in a proportion of infants were assessed independently by two observers, without knowledge of each other's scores, and interobserver agreement was assessed using the FACS reliability formula. ${ }^{4}$

The influence of variables was assessed by analysis of variance and the paired behavioural scores by the $t$ test using the statistical package Minitab. 

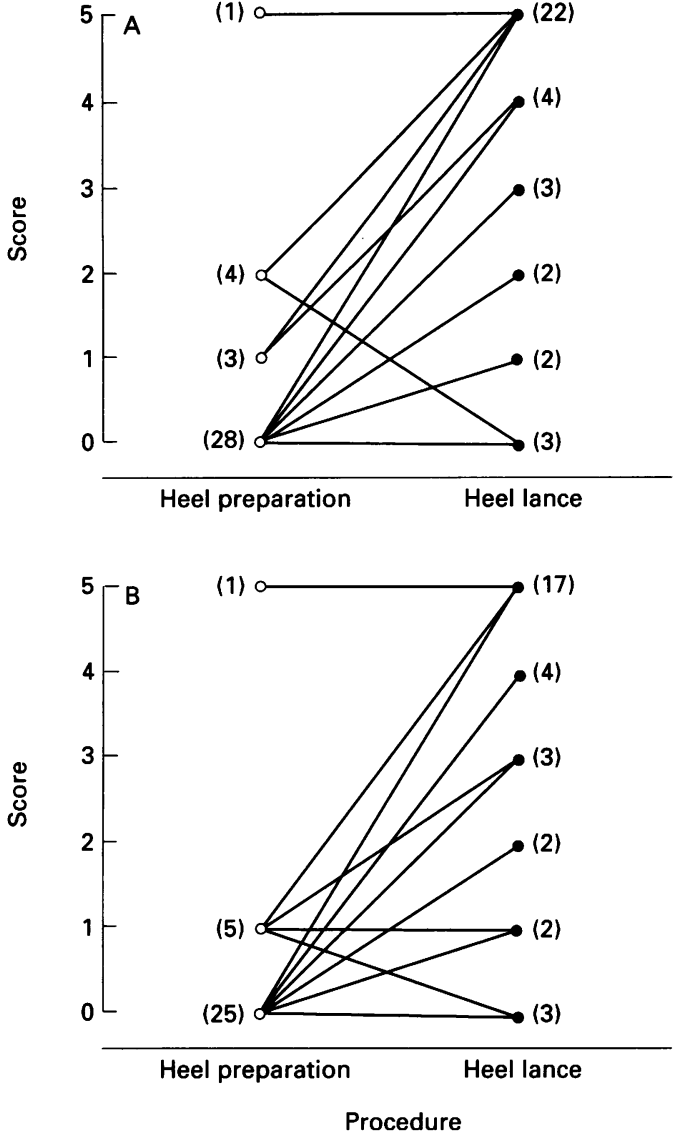

Figure 1 Control and pain scores for $(A)$ term $(n=36)$ and $(B)$ preterm $(n=31)$ infants. Numbers in parentheses are number of patients with each score.

\section{Results}

PATIENT CHARACTERISTICS

Thirty six term ( 23 boys) and 31 preterm (14 boys) infants were studied. The median (range) gestational ages of the infants was 40 (38-42) and $34(32-36)$ weeks respectively, and their ages $4(1-7)$ and 4 (1-17) days. All infants had Apgar scores of 8 or greater at one and five minutes and all had been fed within the two hours before observation.

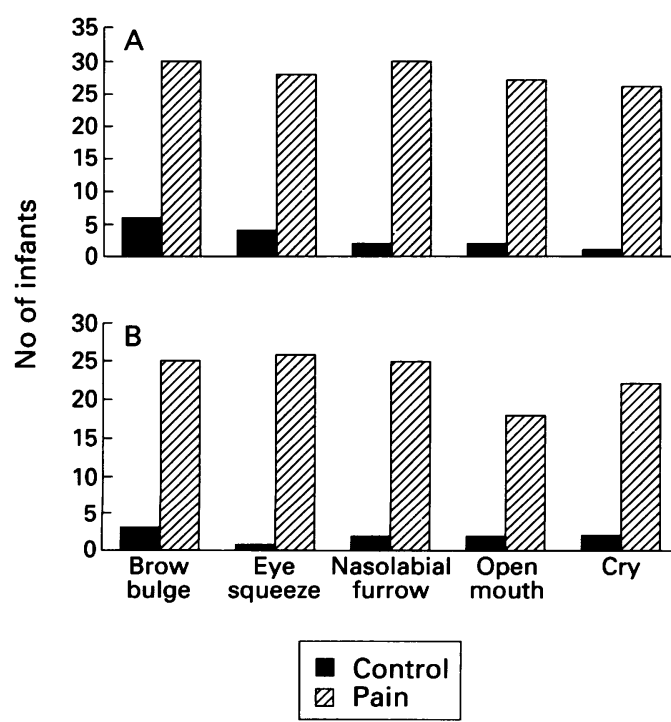

Figure 2 Number of $(A)$ term $(n=36)$ and $(B)$ preterm $(n=31)$ infants showing each behaviour in response to heel preparation and heel lance.
FACIAL ACTION

Term infants

During heel preparation $28(78 \%)$ of the term infants showed none of the facial actions or crying. Only three of these infants remained quiet and expressionless in response to heel lance. Eight $(22 \%)$ infants showed one of the behavioural responses during heel preparation; seven of these showed an increase in the number of responses in response to heel lance. The infant showing no increase in the number of behavioural responses was in Prechtl behavioural state 1 at the time of observation and demonstrated eye squeeze and brow bulge during heel preparation, but no facial action in response to heel lance (fig $1 \mathrm{~A}$ ).

Brow bulge and nasolabial furrow were the facial actions seen most in response to heel lance (30 (83\%) infants). Twenty eight $(78 \%)$ infants showed eye squeeze, 27 $(75 \%)$ open mouth, and $26(72 \%)$ cried (fig 2A).

\section{Preterm infants}

During heel preparation $25(81 \%)$ of the preterm infants showed none of the facial actions or crying; only one showed no change after heel lance. One infant cried and demonstrated all four facial actions in response to heel preparation; he continued to cry and showed the same responses to heel lance. Five (16\%) infants showed only one of the responses to heel preparation; two of these then showed an increase in facial action to heel lance, and two showed no facial actions; one infant was crying at the beginning of observation and continued to do so throughout heel preparation and heel lance (fig 1B).

Eye squeeze was the facial action most often seen in the preterm infants in response to heel lance $(21 / 26,81 \%)$. Twenty five $(81 \%)$ infants showed brow bulge and nasolabial furrow, 18 $(58 \%)$ open mouth, and $22(71 \%)$ cried (fig 2B).

\section{SCORES}

The median (range) control score was $0(0-5)$ and the pain score $5(0-5)$ in the two gestational age groups. There was a median (range) increase of $4(-2$ to 5$)$ in the term infants and 4 ( -1 to 5$)$ in the preterm infants; these increases were statistically significant $(t$ test: $\mathrm{p}<0.001)$.

The control scores were not influenced by gestational age $(p=0.917$ (term); $p=0.873$ (preterm)) or postnatal age $(p=0.269$; $\mathrm{p}=0.231)$, neither were the pain scores influenced by gestation $(p=0.917 ; p=0.907)$ or postnatal age $(p=0.067 ; p=0.415)$.

Female sex, however, was associated with an increased pain score compared with male sex $(p=0.035)$ in the term, but not preterm infants.

The arousal state before sampling did not influence pain scores in either gestational age range group $(p=0 \cdot 108 ; p=0.906)$.

OBSERVER AND TECHNICIAN EFFECTS

Two observers independently assessed 17 infants for the presence or absence of facial action and crying. Using the FACS reliability 
formula there was $94 \%$ agreement between observers.

Six technicians performed the blood tests in the term infants and four in the preterm infants. The median (range) number of blood tests performed by one technician was four (three to seven). No between-technician effect was seen $(p=0 \cdot 311)$.

\section{Discussion}

We have shown that the four most often seen facial expressions in healthy neonates undergoing heel lance can be assessed at the cotside with good interobserver agreement. The frequency of facial action detected by direct observation does not differ greatly from that seen by analysing video recordings which have the advantage of slow playback. ${ }^{4}$

In this group of healthy neonates the response to the painful stimulus was remarkably consistent and was not influenced by gestational age (within each gestational age range group), postnatal age, or arousal. Grunau and Craig, however, have shown that infants who are quietly awake (Prechtl state 4) show significantly more facial actions to heel preparation and heel lance than infants in other states. ${ }^{4}$ The differences between this and the results of our study are not clear; it may simply reflect differences in patient numbers, or it may be influenced by the greater number of facial actions (nine compared with four) assessed by Grunau and Craig. ${ }^{4}$
Our finding that term female infants showed a significant increase in pain score to heel lance compared with male infants is interesting, but did not occur in the preterm group. The marked difference in the number of boys and girls in our term population may explain this effect ( 23 boys, 13 girls). It has been noted previously that boys have shorter latency to crying in response to heel lance than girls, ${ }^{4}$ therefore some subtle sex differences may exist in behavioural responses to painful stimuli.

The consistency of response of healthy neonates to a standard painful stimulus and the reproducibility of results between independent observers suggests that this technique for the assessment of pain can be used as a measure of acute neonatal pain. Its use in longer lasting pain, such as postoperative pain, and its validity in sick infants and those of less than 32 weeks' gestation needs to be validated.

1 Anand KJS, Hickey PR. Pain and its effects in the human fetus and neonate. $N$ Engl $\mathcal{F}$ Med 1987; 317: 1321-9.

2 Johnston CC, Stevens B, Craig KD, Grunau RVE. Developmental changes in pain expression in premature, full-term, two- and four-month-old infants. Pain 1993; 52: 201-8.

3 Craig KD, Whitfield MF, Grunau RVE, Linton J, Hadjistavropoulos HD. Pain in the preterm neonate: behavioural and physiological indices. Pain 1993; 52: 287-99.

4 Grunau RVE, Craig KD. Pain expression in neonates: facial action and cry. Pain 1987; 28: 395-410.

5 Grunau RVE, Johnston CC, Craig KD. Neonatal facial and cry responsiveness to invasive and non-invasive procedures. Pain 1990; 42: 295-300.

6 Johnston CC, Strada ME. Acute pain response in infants: multidimensional description. Pain 1986; 24: 373-82.

7 Prkachin KM. The consistency of facial expressions of pain: a comparison across modalities. Pain 1992; 51: 297-306.

8 Prechtl HFR, Beintema DJ. The neurological examination of the full term newborn infant. Clinics in developmental medicine No 12. London: Hęinemann, 1964. 